\title{
Role of mHealth applications for improving antenatal and postnatal care in low and middle income countries: a systematic review
}

Anam Feroz ${ }^{*}$, Shagufta Perveen and Wafa Aftab

\begin{abstract}
Background: From 1990 to 2015, the number of maternal deaths globally has dropped by 43\%. Despite this, progress in attaining MDG 5 is not remarkable in LMICs. Only $52 \%$ of pregnant women in LMICs obtain WHO recommended minimum of four antenatal consultations and the coverage of postnatal care is relatively poor. In recent years, the increased cellphone penetration has brought the potential for mHealth to improve preventive maternal healthcare services. The objective of this review is to assess the effectiveness of mHealth solutions on a range of maternal health outcomes by categorizing the interventions according to the types of mHealth applications.
\end{abstract}

Methods: Three international online electronic databases were searched between January 1, 2000 and January 25, 2016 to identify studies exploring the role of mHealth solutions in improving preventive maternal healthcare services. Of 1262 titles screened after duplication, 69 potentially relevant abstracts were obtained. Out of 69 abstracts, 42 abstracts were shortlisted. Full text of 42 articles was reviewed using data extraction sheet. A total of 14 full text studies were included in the final analysis.

Results: The 14 final studies were categorized in to five mHealth applications defined in the conceptual framework. Based on our analysis, the most reported use of mHealth was for client education and behavior change communication, such as SMS and voice reminders $[n=9,65 \%]$. The categorization provided the understanding that much work have been done on client education and behavior change communication. Most of the studies showed that mHealth interventions have proven to be effective to improve antenatal care and postnatal care services, especially those that are aimed at changing behavior of pregnant women and women in postnatal period. However, little evidence exists on other type of mHealth applications.

Conclusion: This review suggests that mHealth solutions targeted at pregnant women and women in postnatal period can improve preventive maternal healthcare services. However, there is a need to conduct more controlled-trials and quasi-experimental studies to strengthen the literature in this research area. The review recommends that mHealth researchers, sponsors, and publishers should prioritize the transparent reporting of interventions to allow effective interpretation of extracted data.

Keywords: Mobile health, mHealth interventions, Antenatal care services, Postnatal care services, Low and middle income countries, Systematic review

\footnotetext{
* Correspondence: anam.feroz@aku.edu

Department of Community Health Sciences, The Aga Khan University,

Stadium Road, Karachi 74800, Pakistan
}

(c) The Author(s). 2017 Open Access This article is distributed under the terms of the Creative Commons Attribution 4.0 International License (http://creativecommons.org/licenses/by/4.0/), which permits unrestricted use, distribution, and reproduction in any medium, provided you give appropriate credit to the original author(s) and the source, provide a link to the Creative Commons license, and indicate if changes were made. The Creative Commons Public Domain Dedication waiver (http://creativecommons.org/publicdomain/zero/1.0/) applies to the data made available in this article, unless otherwise stated. 


\section{Background}

There has been an estimated $43 \%$ reduction in the number of maternal deaths globally, from 1990 to 2015 $[1,2]$. The sluggish progress is evident in the current "countdown to 2015" report, which stated that only nine out of 74 countries with the highest Maternal Mortality Rates (MMRs) were on target to meet Millennium Development Goal five (MDG 5) [3]. The reasons for the slow progress in meeting MDG 5 in most countries is related to limited access to preventive maternal health services, poor administration, poor logistical and technical ability, insufficient financial assets and dearth of skilled health personnel [4]. While moving from MDG 5 to Sustainable Development Goals 3 (SDGs), reducing preventable maternal morbidity and mortality still remains a prioritized health agenda.

In low and middle income countries (LMIC), women face a lifetime risk of maternal death of one in 160, as compared with 1 in 3700 for women living in high income countries (HIC) [5]. Most women die because of complications during and following pregnancy including infections, pre-eclampsia, eclampsia and post-partum hemorrhage. Most of these complications are preventable and account for nearly $75 \%$ of all maternal deaths [6].

Antenatal care (ANC) and postnatal care (PNC) interventions have proven to be key health interventions to decrease maternal mortality. Studies in Tanzania and Ethiopia have proven the ability of ANC and PNC to reduce maternal mortality [7-9]. Improving maternal health outcomes requires reinforcement of prevailing evidence-based practices that include World Health Organization (WHO) recommended number of ANC (minimum of four ANC visits) and PNC visits. The timing and optimal number of PNC visits, particularly in resource-limited setting, is an area of debate. However, it is suggested that women should have at least one or more postnatal visits within 2 days of delivery [7, 8]. In LMICs, only $52 \%$ of pregnant women obtain the WHO suggested minimum of four antenatal visits [6]. The coverage of $\mathrm{PNC}$ in $\mathrm{LMIC}$ is relatively poor and, therefore, nearly $40 \%$ of women develop complications following delivery and an almost $15 \%$ encounter potentially life-threatening complications [7, 10]. The poor ANC and PNC attendance account for substantial number of these preventable deaths [11].

Surprisingly, many countries with limited print or internet resources have gained substantial level of cell phone penetration. According to 'International Telecommunication Union 2015' figures, cell phone subscriptions have reached over 7 billion worldwide and in LMICs mobile penetration has reached over $90 \%$. [12, 13]. The increased penetration of cellphone over recent years has brought the potential for mobile health to improve ANC and PNC services by addressing issues such as low literacy level, large geographical distances to services, social marginalization, unskilled human resource, and poor financial resources $[12,13]$. The increasing proliferation of mobile technology is bringing up new opportunities to permit safe, accessible, coordinated and effective maternal health care [6]. There are numerous models of mHealth interventions being used to support pregnant mothers through safe pregnancy and childbirth in LMICs [6, 13]. Mobile health or mHealth, refers to the use of mobile phones, personal digital assistants (PDAs), patient monitoring devices and other Information and Communication Technologies (ICT) to support and deliver health and healthcare services.

Many initiatives have been taken to plot the strength of the evidence regarding mHealth for improving preventive maternal healthcare services including 'Phil Brick's gap analysis for mHealth Alliance', stakeholder interviews, pooled review by Noordam et al., and a systematic review by Tamrat and Kachowski [14-16]. However, none of the reviews explored the effectiveness of mHealth solutions on a range of maternal health outcomes by categorizing the interventions according to the types of mHealth applications. Given the potential significance of the context, a review on exploring the role of mHealth applications for improving antenatal and postnatal care in low and middle income countries may provide valuable insights in to pertinent issues.

Labrique and colleagues identified $12 \mathrm{mHealth}$ applications to respond to various health issues [17]. Few projects involve one application and other projects may include two or more mHealth applications for addressing specific health issue. The classification of 12 mHealth applications as per Labrique and colleagues is illustrated in Table 1. The same framework is used to guide the conduct of this systematic review.

Table 1 Twelve common mHealth applications

1. Client education and behavior change communication (BCC)
2. Sensors and point-of-care diagnostics
3. Registries/ vital events tracking
4. Data collection and reporting
5. Electronic health records
6. Electronic decision support (information, protocols, algorithms,
checklists)
7. Provider to provider communication (user groups and consultation)
8. Provider work planning and scheduling
9. Provider training and education
10. Human Resource management
11. Supply chain management
12. Financial transactions and incentives




\section{Objective}

The objective of this review is to assess the effectiveness of mHealth solutions on a range of maternal health outcomes by categorizing the interventions according to the types of mHealth applications.

\section{Methods}

An electronic systematic literature search was carried out on role of mHealth solutions in improving preventive maternal healthcare services in LMIC. The protocol was registered in the 'International Prospective Register for Systematic Reviews' (PROSPERO) CRD42016035503 [18]. The studies involving target groups of women who were in antenatal and postnatal period and healthcare workers by which mHealth interventions delivered to these groups were included. LMICs were shortlisted according to the World Bank's 2016 Country Classification [19]. Issues regarding use of mobile phones are shared across many LMIC, therefore these studies are more comparable than those conducted in HIC. The inclusion and exclusion criterion applied to this review is illustrated in Table 2.

\section{Information sources and search strategy}

Three international electronic databases PubMed, CINAHL Plus and Cochrane were searched using detailed search strategy. Cross referencing of systematic reviews was also undertaken to identify relevant articles using hand search. The database searches were performed by two researchers independently. The search terms were grouped in to four major categories of interest: population, intervention, outcome and settings. In order to make uniform search terms, the Medical Subject Headings (MeSH) were utilized wherever applicable. The search strategy was also piloted. The search strategy applied to this review is illustrated in Table 3.

\section{Study selection}

The resulting studies were first screened by titles, then by abstract, and finally by full text to progressively eliminate studies not meeting the inclusion criteria. Database searches identified a total of 1290 studies initially. After de-duplication, 1262 records were screened by titles. After title screening, 69 records were screened by abstracts. Full texts of remaining 42 studies were reviewed to determine if they fulfill the inclusion criteria. All the potentially relevant full-texts were assessed for quality using Cochrane Risk of Bias Assessment Tool and Newcastle-Ottawa-Quality Assessment Scale [20, 21]. Two additional relevant studies were also identified from cross-referencing of systematic reviews. Finally, 14 studies were selected and used for the purpose of this review [22-35]. The results of study screening and selection are illustrated in Fig. 1.

\section{Data collection process}

Two reviewers screened the titles, abstracts and full text of selected studies against the inclusion and exclusion criteria independently using a customized data extraction form. Any discrepancies between the two reviewers in this process were discussed with the third reviewer until consensus was reached. The sheet was designed on MS Excel spreadsheet using existing literature and subject experts' opinion. The information extracted included author, year of publication, study title, purpose of study, type of paper/study design, health issue studied, study method, interventional group, control group, primary or secondary intervention for improving preventive

Table 2 Eligibility Criteria

\begin{tabular}{|c|c|c|}
\hline Attribute & Inclusion Criteria & Exclusion Criteria \\
\hline Population & $\begin{array}{l}\text { Studies involving target groups of women in antenatal and } \\
\text { postnatal period and healthcare workers through which } \\
\text { interventions were delivered to these groups. }\end{array}$ & $\begin{array}{l}\text { Studies involving target groups women, adolescent females and girls } \\
\text { over the age of } 5 \text { years who are not pregnant and have not recently } \\
\text { given birth, newborn and children and decision makers and facility } \\
\text { managers that are not directly involved in patient care }\end{array}$ \\
\hline Intervention & Studies involving mHealth interventions & $\begin{array}{l}\text { Studies involving other ICT interventions, ART compliance reminders, } \\
\text { EmONC coverage, managerial and financial level interventions, physical } \\
\text { mobile clinics and teleconsultations }\end{array}$ \\
\hline Outcome & $\begin{array}{l}\text { Outcomes demonstrating improvement in preventive } \\
\text { maternal healthcare services }\end{array}$ & $\begin{array}{l}\text { Outcomes demonstrating skilled birth attendants, emergency care, } \\
\text { quality of life, immunization coverage, cost-effectiveness of intervention, } \\
\text { child development and others }\end{array}$ \\
\hline Setting & Studies conducted or implemented in LMICs & Studies conducted or implemented in high income countries \\
\hline Type of studies & $\begin{array}{l}\text { Original studies, case studies, cross-sectional studies, case control } \\
\text { studies, randomized controlled trials, quasi experimental studies, } \\
\text { before and after (pre-post studies), qualitative formative studies, } \\
\text { systematic reviews and clinical control trials }\end{array}$ & $\begin{array}{l}\text { Commentaries, editorials, symposium proceedings and irretrievable } \\
\text { documents }\end{array}$ \\
\hline Language & Studies available in English Language & Studies which were not available in English translation \\
\hline Time period & Studies published between 1 January, 2000 to 25 January, 2016 & Studies published before 1 January, 2000 and after 25 January, 2016 \\
\hline
\end{tabular}


Table 3 Search Strategy

Population "Pregnancy"[Mesh] OR pregnan* OR pregnant women OR pregnant mother* OR gestational mother* OR women OR maternal age women OR matern* OR "Healthcare providers" [Mesh] AND

Intervention mHealth OR m-Health OR mobile health OR mobile telehealth care OR mobile telemedicine OR mcare OR mobile phone OR mobile devic $^{*}$ OR mobile technology OR mobile commmunication OR satellite phone OR communication satellite OR enterprise digital assistants OR cell phone OR cellular phone OR personal digital assistant* OR PDA OR mobile tablet computers OR smart-phone OR smartphone OR podcast Or pod-cast OR apps OR mobile applications OR text messag* OR short messag* OR short message service OR SMS OR multimedia messag* OR MMS OR texting OR messag* OR text* OR multimedia technol* OR multi-media messag AND

Outcome Reproductive health services OR maternal welfare OR maternal healthcare OR maternal health OR maternal service* OR reproductive health OR reproductive service* OR pre-natal care OR pre-natal visit* OR prenatal care OR prenatal visit* OR antenatal care OR antenatal visit* OR Postnatal Care OR postpartum program* OR safe motherhood OR perinatal complications OR postnatal complication OR perinatal care OR essential preventive maternal health services AND

Setting Developing country OR developing nation OR least developed country OR least developed nation OR less developed nation OR third world country OR third world nation OR under developed country OR remote region OR low and middle income country OR under developed nation OR low and middle income nation OR Armenia OR Moldova OR Bangladesh OR Morocco OR Bhutan OR Myanmar OR Bolivia OR Nicaragua OR Cabo Verde OR Nigeria OR Cameroon OR Pakistan OR Congo, Rep OR Papua New Guinea OR Cote d'Ivoire OR Philippines OR Djibouti OR Samoa OR Egypt, Arab Rep. OR Sao Tome and Principe OR El Salvador OR Senegal OR Georgia OR Solomon Islands OR Ghana OR Sri Lanka OR Guatemala OR Sudan OR Guyana OR Swaziland OR Honduras OR Syrian Arab Republic OR India OR Tajikistan OR Indonesia OR Timor-Leste OR Kenya OR Ukraine OR Kiribati OR Uzbekistan OR Kosovo OR Vanuatu OR Kyrgyz Republic OR Vietnam OR Lao PDR OR West Bank and Gaza OR Lesotho OR Yemen, Rep. OR Mauritania OR Zambia OR Micronesia, Fed. Sts.

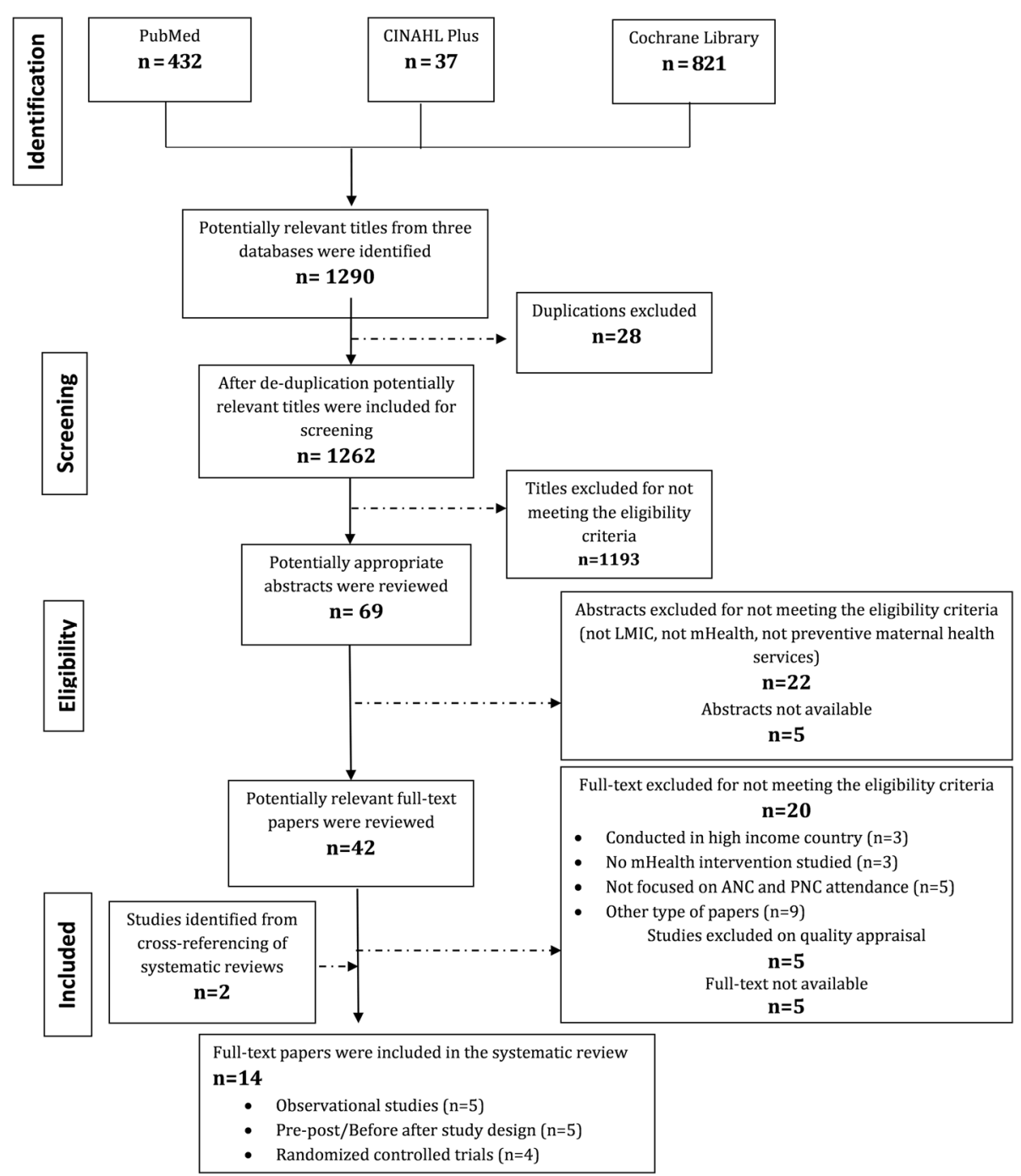

Fig. 1 PRISMA Flow Diagram for Database Search of Studies 
maternal healthcare services, intervention frequency, key study outcomes - improved ANC and PNC services, and quality appraisal of included studies. Later, Labrique and colleagues framework was utilized to organize the data. The summary of included studies on mHealth interventions to improve preventive maternal healthcare services is provided as an appendix [see Additional file 1].

\section{Quality assessment of included studies}

Risk of bias was thoroughly examined for all the included randomized controlled trails (RCTs) $(n=4)$ using the Cochrane Risk of Bias Assessment Tool [20]. This tool was used to evaluate risk of bias in each study by evaluating a study's allocation sequence generation (randomization), allocation concealment, blinding, incomplete data, selective reporting and other potential threats to the study's validity. According to the scale, a low risk of bias is the best possible rank indicating higher quality. All the RCTs $(n=4)$ generally performed well and attained low risk of bias on Cochrane Risk of Bias Assessment Tool.

The quality of non-randomized studies was examined using the 'Newcastle-Ottawa-Quality Assessment Scale' [21]. This scale was used to assess the quality of observational $(n=5)$ and pre-post study design $(n=5)$ by assessing potential sources of bias in the selection and comparability of participants, the assessment of outcomes and the duration and adequacy of follow-up. For non-randomized studies, the scores were awarded out of nine possible points, with higher score indicating higher quality. All the non-randomized studies generally performed well and attained an average score of eight out of nine possible points. Five non-randomized studies attained a score of eight out of nine. Four studies attained a score of seven out of nine. Remaining one study scored five out of nine possible points. The quality assessment of all included studies is provided in the data extract sheet [see Additional file 1].

\section{Results}

The review is stated according to the Preferred Reporting Items for Systematic Reviews and Meta-analyses (PRISMA) guidelines. The data from 14 final studies only fit in to five main mHealth applications defined in the Labrique and colleagues framework which include 'client education and behavior change communication, registries/vital event tracking, data collection and reporting, provider to provider communication, and electronic health records'. All of these applications have been operationalized using various mobile phone functions that include "short message service (SMS), multimedia messaging service (MMS), Interactive voice response (IVR), voice communication, video clips, images, audio clips and packages, apps, mobile phone camera, digital forms and mobile web" [17]. The conceptual framework is adapted to elaborate the potential of mHealth applications for preventive maternal healthcare services (ANC and PNC). The adapted framework is illustrated in Fig. 2.

\section{Types of studies}

Out of fourteen studies which were included, four were randomized controlled trials, five were observational studies (qualitative formative study, cross sectional study) and remaining five were pre-post design studies. All studies included in the review were published within the time period of 2000 to 2016.

\section{Types of mHealth interventions}

The final studies were classified according to the types of mHealth applications. While few studies addressed one mHealth application, many addressed multiple applications. Most of the studies were assigned in more than one category if the intervention was multi-faceted. When the studies were broken down into types of mHealth applications, the total adds up to 16 types of mHealth interventions. The final studies were broadly categorized in five main applications which include client education and behavior change communication $(n=9)$ [22, 23, 26, 28-30, 33-35], registries/vital event tracking $(n=3)$ [31-33], data collection and reporting $(n=2)$ [27, 31], provider to provider communication $(n=1)$ [25], and electronic health records $(n=1)$ [24]. The results of the classification exercise are illustrated in Fig. 3.

\section{Client education and behavior change communication}

In Njoro Division, a randomized controlled trial evaluated the impact of mobile telephone support on antenatal attendance. A group of 191 pregnant women were regularly given advice and prompts regarding pregnancy care and scheduled antenatal visits through mobile phone; whereas, the other groups of 206 pregnant women were provided usual care to continue antenatal visit. Positive association was found among women in intervention group and the number of ANC visits (96.4\% in intervention group and $92.3 \%$ in the control group, $P$ value: 0.002 ) (Fig. 3). [34]

In Zanzibar, a pragmatic cluster randomized controlled trial was conducted in primary health facilities. Twenty-four healthcare facilities were randomized to either intervention group or usual care group. The intervention consists of two components which include SMS and mobile phone voucher. The SMS component was used to send appointment reminders and educational messages to women regarding ANC and PNC. For the SMS component, web-based system was developed to register women with their phone numbers. The mobile phone voucher component allowed pregnant women 


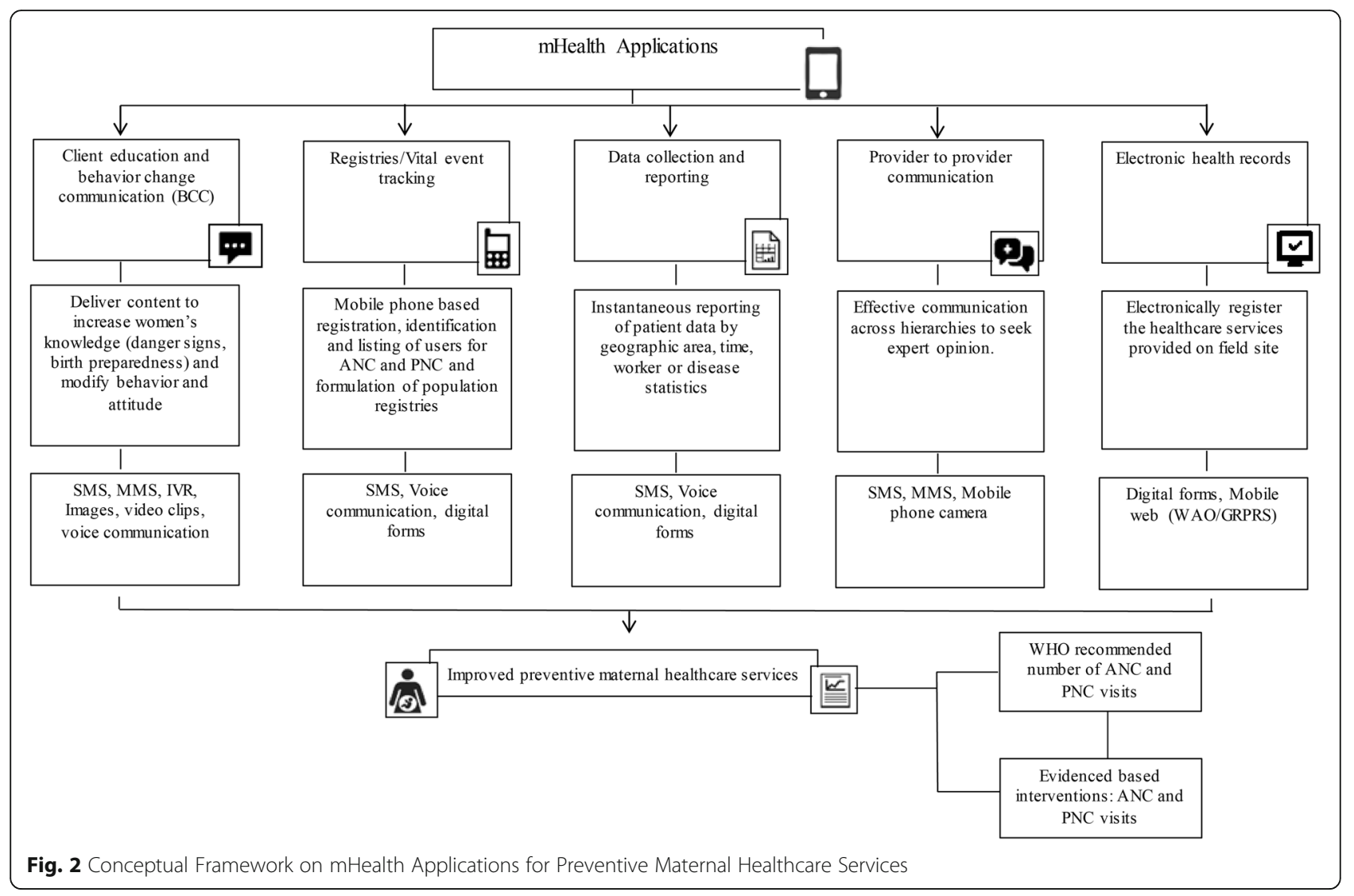

Fig. 2 Conceptual Framework on mHealth Applications for Preventive Maternal Healthcare Services

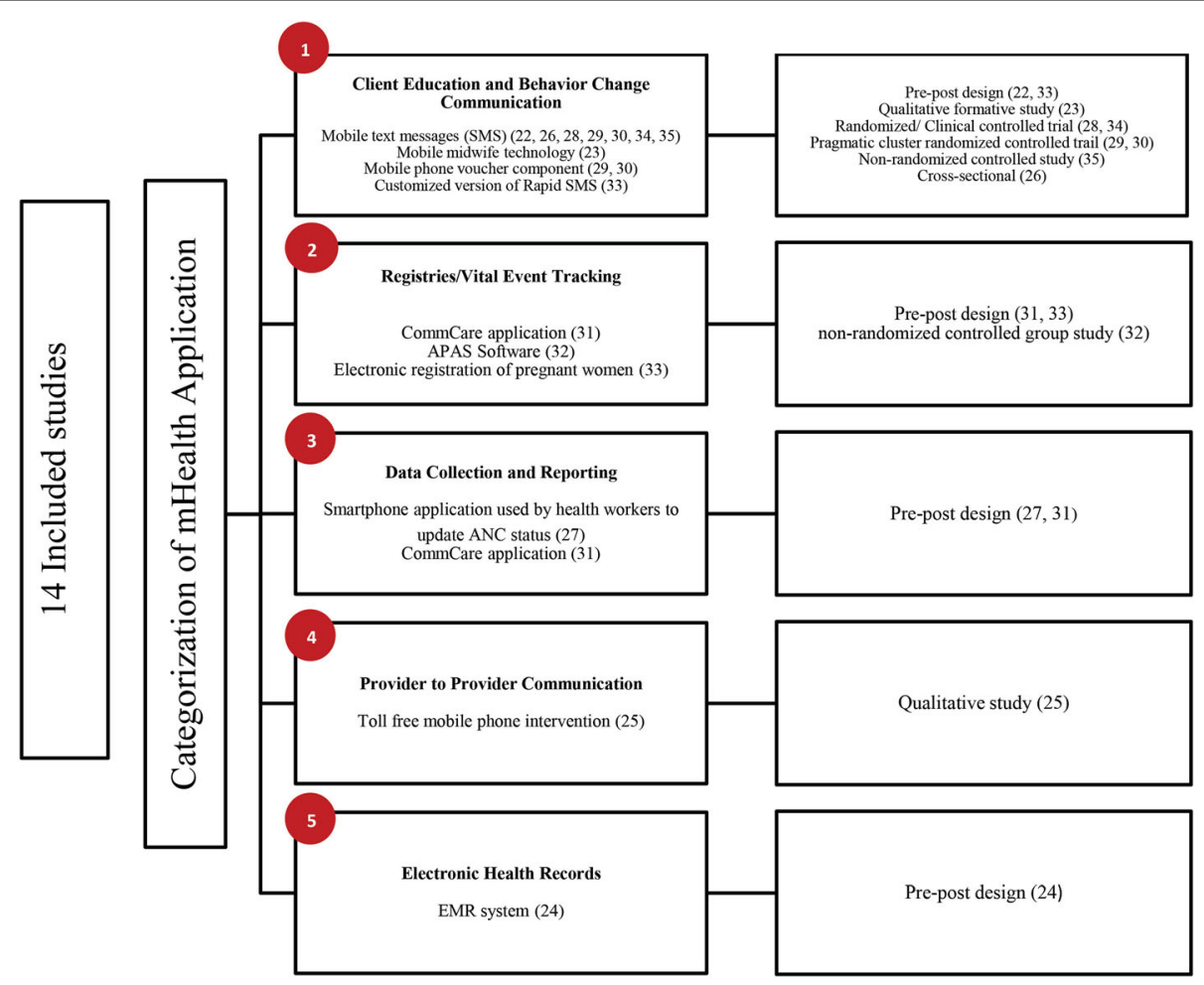

Fig. 3 Classification of the Included Studies Based on the Types of mHealth Interventions Used 
(mothers) to directly communicate with primary healthcare providers. This allowed access to emergency obstetric care through improved communication (Fig. 3). [29]

A Similar pragmatic cluster randomized controlled trial was conducted in primary healthcare facilities of Zanzibar. The primary outcome measure of the trial was four or more ANC visits. The intervention consisted of two components SMS and mobile phone voucher component. The intervention was related with an improvement in ANC visits in the intervention group. In the intervention group, $44 \%$ of women attained four or more ANC visits versus $31 \%$ in the comparison group (OR, 2.39; 95\% CI 1.03-5.55) (Fig. 3) [30].

In Ghana, a qualitative formative study was conducted to explore the role of Mobile Midwife technology in the lives of pregnant mothers. The Mobile Midwife technology routinely sent reminders to pregnant mothers of planned ANC visits and their related significance. Nineteen individual interviews and three Focus Group Discussions (FGDs) were conducted and it was found that women gradually gained trust in the Mobile Midwife technology. Women verbalized that the frequency of ANC visits increased after the introduction of mobile technology (Fig. 3) [23].

In a rural area of Tamil Nadu, India, a pre-post study was conducted to evaluate whether mobile text messaging service is a feasible mode of raising knowledge level regarding Maternal and Child Health $(\mathrm{MCH})$ services. Data was obtained using a questionnaire in three phases; a) baseline assessment, b) intervention: $\mathrm{MCH}$ related messages were sent, c) end line assessment. It was found out that $45(37.5 \%)$ individuals knew about minimum number of antenatal visits during pregnancy after receiving text messages, as compared to $12(10 \%)$ individuals before receiving text messages ( $P$ value $<0.05$, 95\% CI: 0.16-0.38) (Fig. 3) [22].

In Rawanda, a pre-post study was conducted to monitor pregnancy and reduce maternal and child deaths. An innovative SMS- based alert system (RapidSMS-MCH) was developed to permit interactive communication between CHW and mother-infant pairs. A total of 432 CHWs were trained and equipped with mobile phones. A total of 35,734 SMS were sent by CHWs. A total of 11,502 pregnancies were monitored and 362 SMS life threatening events were registered. It was found out that $\mathrm{CHWs}$ were more pro-active in finding new pregnant women and following them up as a result of reminders sent to the mothers (Fig. 3) [33].

In South Africa, a mixed-method study was conducted to increase antenatal awareness and health knowledge by disseminating text messages. First, a controlled trial was conducted in primary health facility of Cape Town. The intervention group $(n=102)$ received SMS and the control group $(n=104)$ received no text messages. The text messages included antenatal health information in their preferred language. A baseline knowledge questionnaire was administered prior to the intervention and after the intervention with additional health related behavior questions. No statistically significant difference was attained between the two groups $(P>0.05)$. A focused group of seven participants from the intervention group was also performed and it was found out that SMS acted as a reminder and a source of motivation for the pregnant mothers (Fig. 3) [28].

In Nigeria, a study was conducted to compare the postnatal Clinic attendance among the intervention group $(n=1126)$ and the historic control group $(n=971)$. Text message reminders were delivered to postnatal mothers in the intervention group. It was found out that the intervention group, receiving text message appointment reminders, were $50 \%$ less likely to fail to attend (FTA) their postnatal appointment (RR of FTA 0.50; 95\% CI, 0.32-0.77; $P=0.002$ ) (Fig. 3) [35].

In Nigeria, a population based survey was conducted to investigate if women with restricted mobile phone access have differential odds of maternal knowledge and service utilization as compared to female mobile phone. Multivariate logistic regressions were used to calculate the odds of maternal knowledge and service utilization by mobile phone usage. Findings showed that women without mobile phone access had significantly lower odds of ANC utilization (OR $=0.48,95 \%$ CI: 0.36-0.64) compared to female mobile phone users. Also, women without mobile phone access had significantly lower knowledge of ANC attendance $(\mathrm{OR}=0.46$, 95\% CI: $0.36-$ $0.59)$ compared to female mobile phone users. No differences were observed by mobile phone users in uptake of postnatal services (Fig. 3) [26].

\section{Registries/vital event tracking}

In Rawanda, a pre-post study was conducted to monitor pregnancy and reduce maternal and child deaths. An innovative SMS-based alert system (RapidSMS-MCH) was developed to ensure Electronic registration of pregnant women through text messages by community health workers (CHWs). $81 \%$ of the annual pregnancies in the district were registered in the system. Reporting compliance among CHWs was $100 \%$ (Fig. 3) [33].

In northern Nigeria, a pre-post study was conducted to assess whether the initiation of the CommCare mobile phone application had an effect on the quality of ANC services provided by lower-level cadre. The CommCare application guides $\mathrm{CHWs}$ to register new clients by collecting demographic information, past medical history, height, weight and other medical information. Also, the application helps in following-up clients on subsequent visits. Through the introduction 
of CommCare, the quality score improved from 13.3 at baseline to 17.2 at end line $(P<0.0001)$ (Fig. 3). [31]

In western Kenya, an evaluation study was conducted to assess the impact of mobile health system on antenatal and postnatal attendance. CHWs $(n=20)$ were interviewed to assess the adherence to ANC and PNC following registration of 800 women in to mobile health system (APAS). All CHWs communicated that APAS help them track vital events efficiently, as compared to paper based tracking system (Fig. 3) [32].

\section{Data collection and reporting}

In northern Nigeria, a before-after study was conducted to assess whether the initiation of the CommCare mobile phone application had an effect on the quality of ANC services provided by lower-level healthcare workers. The CommCare application guides CHWs to collect client data (medical information) in real time. The application helps CHWs to collect information during the examination (fetal heart rate, maternal and fetal danger signs - if any) and lab results (protein or glucose in urine, Hb levels, malaria test, UTI test). Through the introduction of CommCare, the quality score improved from 13.3 at baseline to 17.2 at end line $(P<0.0001)$ (Fig. 3) [31].

In Thai-Myanmar border area, a study was conducted to evaluate the application of mobile phone integrating in to health system to improve ANC for the undeserved population. 'A module containing web-based and mobile technology system was developed to generate ANC visit schedule dates in which CHW could cross-check, identify and update the mother's ANC status at the healthcare facility or at the household location when performing home visit'. Findings showed that the module enhanced ANC coverage in the Thai Myanmar border area by developing better procedures of data collection and reporting (Fig. 3) [27].

\section{Provider to provider communication}

In rural Bangladesh, a qualitative research was conducted to assess the impact of toll free mobile communication in improving communication for maternal and neonatal complication. Focused group discussions, in-depth interviews and semi-structures interviews were conducted with community skill birth attendants and pregnant women. Women verbalized that once a complication is reported over phone, SBAs either visit to mothers by themselves or advise them for direct referral. More than $80 \%$ SBAs communicated with solution linked group to receive prompt help. The solution linked group involved eight experts from the field of maternal and child health. Women verbalized that SBAs have become competent in managing complications due to effective communication with solution linked group (Fig. 3) [25].

\section{Electronic health records}

In rural Kenya, a study implemented a 'novel cloudbased electronic medical record system' in a $\mathrm{MNCH}$ outpatient setting and assessed its impact on improving completeness of data collected by healthcare staff. Findings showed significant improvements in completeness of the antenatal record were reported through implementation of Electronic Medical Records (EMR) -based data verification (Fig. 3) [24].

\section{Type of outcomes examined \\ Antenatal care services}

Four studies examining ANC services were RCTs [2830, 34]. All studies used text message (SMS) appointment reminders and antenatal education for pregnant women and two also provided the women with mobile phone voucher component to contact their health worker, if needed [29,30]. All studies found a positive association between women in intervention group receiving mHealth intervention and the number of ANC visits. Five studies examined ANC services before and after implementation of mHealth applications for improved patient electronic records, data collection and reporting, vital event tracking and automated appointment reminders; these studies similarly found a statistically significant improvement in on-time ANC attendance, data completeness, vital event tracking and registries following implementation [22, 24, 27, 31, 33]. Two qualitative studies conducted in LMICs evaluated the impact mobile midwife technology and toll free mobile phone communication on quality of ANC and health advice; women in both studies verbalized that ANC visits have improved after the introduction of mobile technology and SBAs have become competent in managing complications due to communication with 'solution linked group' [23, 25]. The remaining two studies examining ANC services found some selfreported behavior change from both patients and health workers after using APAS software and RapidSMS application [26, 32].

\section{Postnatal care services}

Only few studies evaluated the impact of mHealth interventions on postnatal care services [26, 32, 35]. One study examined the effect of mHealth intervention on PNC attendance by comparing an intervention group with a historical control group and found that the intervention group, receiving text message appointment reminders, were $50 \%$ less likely to fail to attend their visit $(P=0.002)[35]$. 


\section{Discussion}

All included studies showed some evidence that mHealth interventions can play a major role in improving a range of maternal health outcomes. Most of the studies took place in East Asia and Sub-Saharan Africa, while some were undertaken in Middle East and South Asia. mHealth solutions are increasingly being utilized to increase the quality of pre- and post-pregnancy care, and as a way of collecting pregnancy data. A few studies reported that mHealth interventions, particularly those delivered through SMS, were associated with improved utilization of preventive maternal healthcare services, including uptake of recommended ANC and PNC services. In most studies, authors did not describe the basis of their intervention, in terms of its pathways through which it would be delivered to target groups. Moreover, the studies did not utilize a common taxonomy for explaining the type and purpose of the mHealth intervention. In addition, several studies combined multiple mHealth interventions [31, 33], make it difficult to determine to what extent each intervention brought the expected outcome.

To aid interpretation, Labrique and colleagues framework was adapted for categorizing the mHealth interventions according to their purpose, as previously described (Fig. 1). Based on our analysis, the most reported use of mHealth was for client education and behavior change communication, such as SMS reminders, [22, 23, 26 28, 29, 30, 33 34, 35]. This was followed by registries and vital event tracking, to enable ANC and PNC registration or reporting of health indicators [31-33]. The other observed categories were mHealth as a Data collection and reporting, mainly to ensure data recording and completeness [27, 31]; as a provider to provider communication platform, to access support from care providers [25]; and as an electronic health record system to ensure availability of health information systems [24]. The categorization of the studies in to various mHealth applications provided the understanding that the strongest evidence exists on client education and behavior change communication mHealth application. However, little evidence exists on other type of mHealth applications such as, registries and vital events tracking, data collection and reporting, provider to provider communication and electronic health records. Thus, there is an extensive need to perform research in overlooked areas to strengthen the evidence base.

This review with comprehensive search strategy, analyses both ANC and PNC attendance indicators as two most important components of preventive maternal healthcare services. However, much of the literature reported impact of mHealth solutions on antenatal attendance and only a few assessed the effect of mHealth interventions on postnatal attendance. ANC and PNC, both have long been regarded as critical for detecting a number of women who are at more risk of poor pregnancy outcomes [8]. Especially, a strong positive association between improved PNC services and reduction in maternal mortality is established. As improved PNC services prevent women from life-threatening fatal pathologies (post-partum hemorrhage, post-partum depression, etc.). It is advocated that PNC should be given importance by using mHealth solutions to identify post-pregnancy risks in women who may be predisposed to a range of potentially fatal pathologies.

As with most systematic reviews in the field of $\mathrm{mHealth}$, this review is restricted by the difficulty of analyzing complex intervention studies and the variable description of interventions across studies. More studies are needed to refine the current work with a larger body of evidence and to establish how best to integrate it with the published existing frameworks. The heterogeneity of the interventions and study outcomes restricted the interpretation through meta-analyses. The current literature contains many studies describing the use of mHealth for improving preventive maternal healthcare services in LMICs but only some have vigorously assessed the impact of mHealth solutions. Overall, most studies included in this review was of moderate quality, highlighting the significance of increasing the methodological rigor of future research. For randomized trials, there is need for allocation concealment and adequate blinding of outcomes, while the quality of observational studies can be improved via prospective research designs and adjustment for confounding variables.

As a result of methodological limitations and the small number of studies meeting the inclusion criteria, further randomized controlled trials and scaling up of pilot studies are needed to explore the potential of mHealth for improving preventive maternal healthcare services.

\section{Conclusion}

The review concludes that mHealth solutions targeted at pregnant women and women in postnatal period can improve antenatal and postnatal care services in LMICs. There is a growing body of evidence highlighting the effectiveness of mHealth interventions on a range of maternal health outcomes in LMIC, but overall the available literature is weak and the results, in most cases, are too inconsistent to enable robust conclusions to be drawn about impacts. However some supportive literature exists with respect to the use of SMS for increasing ANC and PNC services. In particular, RCTs with economic, clinical and long-term patient-centered outcomes are suggested. This review recommends that mHealth researchers, sponsors, and publishers should prioritize the transparent reporting of interventions in terms of their aims, contexts, pathway of delivery and assumed 
mechanisms of impact to allow effective interpretation of extracted data. As low cost smartphones start to penetrate in these regions, a new generation of mobile applications are now emerging, which will also require evidence-based methods to establish their safety, efficacy and societal impacts.

\section{Additional file}

Additional file 1: Summary of study findings. Description of included studies and quality appraisal (DOCX $21 \mathrm{~kb}$ )

\section{Abbreviations}

ANC: Antenatal care; ART: Antiretroviral Therapy; CHWs: Community Health Workers; EmONC: Emergency Obstetric and Neonatal Care; EMR: Electronic Medical Records; FGDs: Focus Group Discussions; HIC: High Income Countries; ICT: Information and Communication Technologies; IVR: Interactive Voice Response; LMIC: Low and Middle Income Countries; MCH: Maternal and Child Health; MDG: Millennium Development Goal; MeSH: Medical Subject Headings; MMR: Maternal Mortality Rate; MMS: Multimedia Messaging Service; PDA: Personnel Digital Assistants; PNC: Postnatal Care; PRISMA: Preferred Reporting Items for Systematic Reviews and Meta-analyses; RCTs: Randomized Control Trials; SBA: Skilled Birth Attendants; SDGs: Sustainable Development Goals; SMI: Safe Motherhood Initiative; SMS: Short Messaging Service; WHO: World Health Organization

\section{Acknowledgements}

The authors would like to thank Dr. Fauziah Rabbani, Professor and Chair, Department of Community Health Science, The Aga Khan University, Pakistan, for her technical assistance in developing literature search criteria and for providing overall guidance during initial planning of this systematic review.

\section{Funding}

Not applicable.

\section{Availability of data and materials}

The datasets during and/or analyzed during the current study available from the corresponding author on reasonable request. Most of the data generated or analyzed during this study are included in this published article [and its supplementary information files].

\section{Authors' contributions}

The authors involved in this review are public health graduates with expertise in health policy and health systems research. AF made substantial contributions to conception and design of the systematic review. AF was involved in drafting the manuscript. WA and SP reviewed several drafts of this paper. WA and SP were involved in critically revising the manuscript for important intellectual content. All authors have read and approved the final manuscript.

\section{Ethics approval and consent to participate}

Not applicable.

\section{Consent for publication}

Not applicable.

\section{Competing interests}

The authors declare that they have no competing interests.

\section{Publisher's Note}

Springer Nature remains neutral with regard to jurisdictional claims in published maps and institutional affiliations.
Received: 28 October 2016 Accepted: 2 November 2017

Published online: 07 November 2017

\section{References}

1. Unicef. Trends in maternal mortality: 1990 to 2013. 2014. Retrieved from http://www.who.int/reproductivehealth/publications/monitoring/ maternal-mortality-2013/en/

2. World Health Organization, UNICEF. Trends in maternal mortality: 1990 to 2013: estimates by WHO, UNICEF, UNFPA, the World Bank and the United Nations population division: executive summary. 2014

3. Finlayson K, Downe S. Why do women not use antenatal services in lowand middle-income countries? A meta-synthesis of qualitative studies. PLoS Med. 2013:10(1):e1001373.

4. Bazile J, Rigodon J, Berman L, Boulanger VM, Maistrellis E, Kausiwa P, Yamin AE. Intergenerational impacts of maternal mortality: qualitative findings from rural Malawi. Reprod Health. 2015;12(1):1.

5. Watterson JL, Walsh J, Madeka I. Using mHealth to improve usage of antenatal care, postnatal care, and immunization: A systematic review of the literature. BioMed research international. 2015;25:2015.

6. World Health Organization. World health statistics 2012. Geneva, Switzerland: World Health Organization; 2012.

7. Langlois ÉV, Miszkurka M, Zunzunegui MV, Ghaffar A, Ziegler D, Karp I. Inequities in postnatal care in low-and middle-income countries: a systematic review and meta-analysis. Bull World Health Organ. 2015;93(4):259-70G.

8. Mrisho M, Obrist B, Schellenberg JA, Haws RA, Mushi AK, Mshinda H, Tanner M, Schellenberg D. The use of antenatal and postnatal care: perspectives and experiences of women and health care providers in rural southern Tanzania. BMC pregnancy and childbirth. 2009;9(1):1.

9. Regassa N. Antenatal and postnatal care service utilization in southern Ethiopia: a population-based study. Afr Health Sci. 2011;11(3)

10. Comfort $A B$, Peterson $L A$, Hatt LE. Effect of health insurance on the use and provision of maternal health services and maternal and neonatal health outcomes: a systematic review. J Health Popul Nutr. 2013;31(4 Suppl 2):S81.

11. Nurmatov UB, Lee SH, Nwaru BI, Mukherjee M, Grant L, Pagliari C. The effectiveness of mHealth interventions for maternal, newborn and child health in low-and middle-income countries: protocol for a systematic review and meta-analysis. J Glob Health. 2014;4(1):010407.

12. UCL Public Policy. mHealth: can mobile technology improve health in lowand middleincome countries. 2015. Retrieved from https://www.ucl.ac.uk/ public-policy/for-policy-professionals/research-insights/mhealth_briefing.pdf.

13. Eysenbach G. CONSORT-EHEALTH: improving and standardizing evaluation reports of web-based and mobile health interventions. J Med Internet Res. 2011;13(4):e126

14. PHilbriCk WC. mHealth, MNCH: State of the Evidence. Trends, gaps, stakeholder needs, and opportunities for future research on the use of mobile technology to improve maternal, newborn, and child health. Washington: UN Foundation; 2013.

15. Noordam AC, Kuepper BM, Stekelenburg J, Milen A. Improvement of maternal health services through the use of mobile phones. Tropical Med Int Health. 2011;16(5):622-6.

16. Tamrat T, Kachnowski S. Special delivery: an analysis of mHealth in maternal and newborn health programs and their outcomes around the world Matern Child Health J. 2012;16(5):1092-101.

17. Labrique $A B$, Vasudevan $L$, Kochi E, Fabricant $R$, Mehl G. mHealth innovations as health system strengthening tools: 12 common applications and a visual framework. Global Health: science and. Practice. 2013;1(2):160-71

18. Feroz A, Perveen $S$, Aftab W. Role of mHealth solutions in improving antenatal and postnatal care attendance in low and middle income countries: a systematic review. 2016. PROSPERO 2016:CRD42016035503 Available from http://www.crd.york.ac.uk/PROSPERO/display_record.asp?ID= CRD42016035503

19. World Bank. Country and lending groups. 2016. Retrieved from http://data. worldbank.org/about/country-and-lending-groups站ower_middle_income.

20. Green S, \& Higgins JPT. Cochrane handbook for systematic reviews of interventions version 5.1. 0. The Cochrane Collaboration. 2011. Retrieved from http://www.cochrane-handbook.org/.

21. Wells GA, Shea B, O'connell D, Peterson JE, Welch V, Losos M, Tugwell P. The Newcastle-Ottawa scale (NOS) for assessing the quality of nonrandomised studies in meta-analyses. 2000. Retrieved from http://www.evidencebased publichealth.de/download/Newcastle_Ottowa_Scale_Pope_Bruce.pdf. 
22. Datta SS, Ranganathan P, Sivakumar KSA. Study to assess the feasibility of text messaging service in delivering maternal and child healthcare messages in a rural area of Tamil Nadu, India. Australas Med J. 2014;7(4):175.

23. Entsieh $\mathrm{AA}$, Emmelin $\mathrm{M}$, Pettersson $\mathrm{KO}$. Learning the $\mathrm{ABC}$ s of pregnancy and newborn care through mobile technology. Glob Health Action. 2015;8

24. Haskew J, Rø G, Saito K, Turner K, Odhiambo G, Wamae A, Sharif S, Sugishita T. Implementation of a cloud-based electronic medical record for maternal and child health in rural Kenya. Int J Med Inform. 2015;84(5):349-54.

25. Huq NL, Azmi AJ, Quaiyum MA, Hossain S. Toll free mobile communication: overcoming barriers in maternal and neonatal emergencies in rural Bangladesh. Reprod Health. 2014;11(1):1.

26. Jennings L, Omoni A, Akerele A, Ibrahim Y, Ekanem E. Disparities in mobile phone access and maternal health service utilization in Nigeria: a population-based survey. Int J Med Inform. 2015;84(5):341-8.

27. Kaewkungwal J, Singhasivanon P, Khamsiriwatchara A, Sawang S, Meankaew P, Wechsart A. Application of smart phone in" better border healthcare program": a module for mother and child care. BMC medical informatics and decision making. 2010;10(1):1.

28. Lau YK, Cassidy T, Hacking D, Brittain K, Haricharan HJ, Heap M. Antenatal health promotion via short message service at a midwife obstetrics unit in South Africa: a mixed methods study. BMC pregnancy and childbirth. 2014;14(1):1.

29. Lund S, Hemed M, Nielsen BB, Said A, Said K, Makungu MH, Rasch V. Mobile phones as a health communication tool to improve skilled attendance at delivery in Zanzibar: a cluster-randomised controlled trial. BJOG Int J Obstet Gynaecol. 2012;119(10):1256-64.

30. Lund S, Nielsen BB, Hemed M, Boas IM, Said A, Said K, Makungu MH, Rasch V. Mobile phones improve antenatal care attendance in Zanzibar: a cluster randomized controlled trial. BMC pregnancy and childbirth. 2014;14(1):1.

31. McNabb M, Chukwu E, Ojo O, Shekhar N, Gill CJ, Salami H, Jega F. Assessment of the quality of antenatal care services provided by health workers using a mobile phone decision support application in northern Nigeria: a pre/post-intervention study. PLoS One. 2015;10(5):e0123940.

32. Mushamiri I, Luo C, liams-Hauser C. Amor YB. Evaluation of the impact of a mobile health system on adherence to antenatal and postnatal care and prevention of mother-to-child transmission of HIV programs in Kenya. BMC Public Health. 2015;15(1):1.

33. Ngabo F, Nquimfack J, Nwaigwe F, Mugeni C, Muhoza D, Wilson DR, Kalach J, Gakuba R, Karema C, Binagwaho A. Designing and implementing an innovative SMS-based alert system (RapidSMS-MCH) to monitor pregnancy and reduce maternal and child deaths in Rwanda. Pan African Medical Journal. 2012;13:31.

34. Fedha T. Impact of mobile telephone on maternal health service care: a case of Njoro division. Open Journal of Preventive Medicine. 2014;2014

35. Adanikin Al, Awoleke JO, Adeyiolu A. Role of reminder by text message in enhancing postnatal clinic attendance. Int J Gynecol Obstet. 2014;126(2):179-80.

\section{Submit your next manuscript to BioMed Central and we will help you at every step:}

- We accept pre-submission inquiries

- Our selector tool helps you to find the most relevant journal

- We provide round the clock customer support

- Convenient online submission

- Thorough peer review

- Inclusion in PubMed and all major indexing services

- Maximum visibility for your research

Submit your manuscript at www.biomedcentral.com/submit 